\title{
EMITTANCE CHARACTERISTICS OF NEGATIVE ION BEAMS GENERATED BY THE SPUTTER TECHNIQUE'
}

\author{
G. D. Alton \\ Oak Ridge National Laboratory \\ P. O. Box 2008 \\ Oak Ridge, Tennessee 37831-6368
}

DE89 015866

Average emittance data for ion beams extracted from cesium-sputter negative ion sources equipped with spherical, ellipsoidal, and cylindrical geometry cesiumsurface ionizers are presented. The attributes of the respective source geometries are described in terms of their cesium ion optical properties. The results of recent measurement of the emittances of momentum-analyzed beams extracted from the ellipsoidal geometry source are also presented. These measurements indicate the presence of a species-dependent effect. The effect is believed to be attributable to differences in the energy spreads of the respective negative ion beams introduced by the sputter generation process.

\section{DISCLAIMER}

This report was prepared as an sccount of work sponsored by an agency of the United States Government. Neither the United States Government nor any agency thereof, nor any of their employees, makes any warranty, express or implied, or assumes any legal liability or responsibility for the accuracy, completeness, or usefulness of any information, apparatus, product, or process disclosed, or represents that its use would not infringe privately ownod rights. Reference herein to any specific commercial product, process, or service by trade name, trademark, manufacturer, or oherwise does not necessarily constitute or imply its endorsement, recommendation, or favoring by the United States Government or any agency thereof. The views and opinions of authors expresued herein do not necessarily state or reflect those of the United States Government or any agency thereof.

- Research sponsored by the U.S. Department of Energy under contract DE-AC05840R21400 with Martin Marietta Energy Systems, Inc.

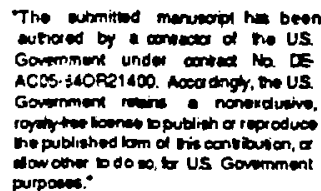




\subsection{INTRODUCTION}

The sputter technique has proved to be a universal method for generating atomic and molecular negative ion beams from chemically active elements. In addition to being versatile in terms of species, sources based on this concept, such as those described in Refs. 1 and 2, are simple in design, easy to operate, and generally have long lifetimes. Because of these factors, such sources are utilized extensively in most tandem electrostatic accelerator heavy ion physics research laboratories, as well as for use in a growing number of other applications, including high-energy ion implantation and tandem accelerator mass spectrometry. In such sources, the sample material is mounted on a biased probe which is maintained at a negative potential of $1-5 \mathrm{kV}$ with respect to the housing in a controlled flux of a neutral Group IA element, usually cesium, which effects a lowering of the work function and enhances the probability for negative ion formation during the sputter ejection process. [3] Sputtering of the sample material is effected by bombardment with positive Group IA element ions which are formed by direct surface ionization of atoms during collisions with a hot, high-work-function ionizer. Negative ion sources based on the sputter principle all share in common the influences of the intrinsic energy and angular distribution characteristics of the sputter process on the emittances of extracted ion beams.

The quality of a particle beam is usually measured in terms of the emittance $\varepsilon$ and brightness $B$ of the beam. These quantities are figures of merit which are frequently used to determine the compatibility of beams with a given beam transport system and to predict their behavior in a particular ion optical system. These quantities are related to each other through the ion beam intensity $I$ and their 
significance is a direct consequence of Liouville's theorem. This fundamental theorem states that the motion of a group of particles, under the action of conservative force fields, is such that the local number density in the six-dimensional phase space volume $\left(x y z p_{x} p_{y} p_{z}\right)$ everywhere remains constant. An ion beam can be represented by a group of points, all of which lie within a six-dimensional hypervolume. The effective phase space volume of an ion beam may increase due to interactions with residual gas particles and/or gas and foil strippers, beam transport and acceleration system aberrational effects, and space charge effects, but can never be less than the value which occurs immediately following extraction from the source. Thus, the magnitude of the phase space volume or a quantity proportional to the phase space volume (emittance) measured immediately following extraction is a unique characteristic of the particular ion source.

\subsection{NEGATIVE ION SOURCES BASED ON DIRECT SURFACE IONIZATION OF CESIUM VAPOR}

Sputter-type sources can be categorized according to the means for producing the positive ion beam used to sputter the sample. Several sources utilize direct surface ionization of cesium vapor as it comes in contact with a hot, high-work-function surface to form positive ions which are then accelerated against a negatively biased probe containing the material of interest. The positive ion currents achievable in such sources can attain values up to the space charge limit of the particular electrode configuration. The space charge limited current $1+$ which can be accelerated through a potential difference $V$ between the sample and surface ionizer is given by: 


$$
1^{+}=P V 3 / 2
$$

where $P$ is the perveance of the particular electrode configuration. The perveance is a function of the geometry of the electrode system and mass $M_{1}$ of the bombarding ion species. The perveance can be calculated by the use of computer programs, such as described in Refs. 4 and 5, which solve Poisson's equation for the particular electrode system.

State-of-the-art negative ion sources based on direct surface ionization of cesium are described below. All of the sources were designed and developed at the Oak Ridge National Laboratory.

\subsection{The Source Equipped with a Spherical Geometry lonizer}

The space-charge-limited optics for cesium ion flow in a source equipped with a spherical geometry ionizer is shown schematically in Fig. 1. This source has been described previously [6] and the emittance of the source is reported in Ref. 7. The positive cesium ion beam current density distribution at impact with the sample surface is typically $\sim 0.75-\mathrm{mm}$ full diameter when the sample is positioned at the focal point of the system. The computed cesium ion current density distribution at impact with the sample surface is shown in Fig. 2. The computed perveance for cesium in this electrode configuration is $P=2 \times 10^{-9}\left[A / V^{3 / 2}\right]$. 
2.2 The Source Equipped with an Ellipsoidal Geometry Cesium Ionizer

This source is briefly described and the emittances given in Ref. 8 . Figure 3 illustrates the optics for space-charge-limited cesium flow in this highly converging electrode system, while the computed cesium ion current density distribution on the sample surface is shown in Fig. 4. (In practice, the sample wear pattern is observed to have a diameter $\hat{\emptyset} \cong 1.25 \mathrm{~mm}$ because of the difficulty of precisely placing the sample at the theoretical focal point of the electrode system.) This electrode configuration has a high perveance in relation to other focusing systems. The computationally determined perveance for the electrode system is found to be $P=17 \times 10^{-9}\left[\mathrm{~A}^{3 / 2}\right]$.

2.3 The Source Equipped with a Cylindrical Geometry Ionizer

The cylindrical geometry ionizer source configuration shown in Fig. 5 has been described in detail in Refs. 6 and 9 and the emittance and brightness characteristics of the source have been reported in Refs. 7 and 8 . The observed wear pattern from this source is composed of two parts: a region of concentrated wear with full diameter of $\sim 0.75 \mathrm{~mm}$, and a low-density, uniform-wear region with a diameter of $\sim 4.5 \mathrm{~mm}$. Computer simulation of the cesium ion beam optics, such as shown in Fig. 6, agree almost in detail with the observed wear patterns on the sample. Because of the greater size of the region of negative ion generation, the emittance of this source is expected to be larger than those of the previously described sources equipped with spherical and ellipsoidal geometry ionizers. The computed perveance of this ionizer geometry for cesium is $P \cong 57 \times 10^{-9}\left[\mathrm{~A}^{3}{ }^{3 / 2}\right]$. 


\subsection{EMITTANCE MEASUREMENTS}

\subsection{Emittance Definitions}

For ion beam transport, the transverse components of phase space are usually the most important. If the transverse components of motion of a group of particles are independent in configuration space, the motion of the particles in the orthogonal planes $\left(x, p_{x}\right),\left(y, p_{y}\right)$, and $\left(z, p_{z}\right)$ will be uncoupled and, therefore, the phase spaces associated with each of these planes will be separately conserved. The emittance $\varepsilon$ of an ion beam is proportional to the transverse phase space and thus is also a conserved quantity. The emittance definitions used in this report are given by the following relations:

$$
\begin{aligned}
& \varepsilon_{n y}=\pi\left(\iint \frac{\mathrm{dxdx^{ \prime }}}{\pi}\right) \sqrt{\mathrm{E}_{1}}(\mathrm{x} \text {-direction }), \\
& \varepsilon_{\mathrm{ny}}=\pi\left(\iint \frac{\mathrm{dyd} \mathrm{y}^{\prime}}{\pi}\right) \sqrt{\mathrm{E}_{1}}(\mathrm{y} \text {-direction }), \\
& \text { and } \varepsilon=\left(\varepsilon_{n x}, \varepsilon_{n y}\right)^{1 / 2} .
\end{aligned}
$$

In Eqs. $2, x, y$ are position coordinates, $x^{\prime}, y^{\prime}$ are angular coordinates, and $E_{1}$ is the energy of the ion beam. Emittance in this prescription is usually given in units of $\pi$ $\mathrm{mm} \cdot \operatorname{mrad}(\mathrm{MeV})^{1 / 2}$. 
4.2 The Experimental Apparatus

Emittance measurements of the ion beams extracted from the sources, described previously, were determined by use of the equipment and procedures described in Refs. 7 and 8. The scurces were mounted on a test stand capable of accelerating the negative ion beams to energies of $30 \mathrm{keV}$ with energies of $20 \mathrm{keV}$ typical of all measurements; a conventional, three-cylinder einzel lens was used to focus the ion beam onto the object point of a double-focusing $90^{\circ}$ magnet with beriding radius $\rho=0.61 \mathrm{~m}$. Negative ion beams were extracted from the source at a potential difference maintained between the sputter probe and the cesium surface ionizer, typically $1-5 \mathrm{kV}$, postaccelerated to a final energy of $20 \mathrm{keV}$, and focused by means of the einzel lens onto the object plane of the magnet. lons of the desired mass were then deflected through $90^{\circ}$ and focused at the image point of the mass analysis system. The intensity of the negative ion beam was monitored prior to and following momentum analysis by insertion of remotely actuatable Faraday cups; the Faraday cups are located immediately behind variable slit-type apertures which are positioned at the object and image planes of the momentum analysis system. With the Faraday cups removed, the beam passed onto the slit detector unit of the emittance measurement device, described in detail previously, $[6,7]$ where emittance measurements were made. 


\subsection{EXPERIMENTAL RESULTS}

\subsection{Comparative Average Emittance Data}

li we ignore the presence of intensity or species dependent effects and obtain a weighted average of all available emittance contour data, we obtain the average normalized emittance $\varepsilon$ versus percentage of total average negative ion beam intensity data shown in Fig. 7 for the sources described previously. The average normailzed emittances $\varepsilon$ of the spherical, ellipsoidal, and cylindrical sources at the $80 \%$ beam intensity contour are, respectively, $\sim 6.3, \sim 8.0$, and $8.7 \pi \mathrm{mm} . \mathrm{mrad}(\mathrm{MeV})^{1 / 2}$.

\subsection{Species-Dependent Emittance Effect}

From earlier investigations, [8] it was also noted that the emittances of $\mathrm{Si}^{-}$ negative ion beams were consistently $15-20 \%$ lower than ion beams such as $\mathrm{Ni}^{-}$and Au-. This finding was more pronounced for ionizer geometries which sharply focused the cesium ion beam onto the sample surface. However, this effect was not clearly delineated in these early experiments. This observation prompted efforts (reported in detail in Refs. 10 and 11) to look more carefully for the effect with the ellipsoidal geometry source which focuses the cesium ion beam to a well-defined region of the sample surface. 
The investigations were performed at precisely the same inn source operational parameters for each of the samples (cesium oven temperature $\left(196^{\circ} \mathrm{C}\right.$ ), sputter probe voltage with respect to housing $(-3 \mathrm{kV})$, ion extraction voltage $(-20 \mathrm{kV})$, and sample position with respect to the cesium surface ionizer). Negative ion beams from each of the samples were extracted from the source, further accelerated to a final energy of 20 $\mathrm{keV}$, and transported through the ion source test facility discussed previously. Emittance measurements were then made utilizing the hardware, procedures, and data analysis techniques described in Refs. 7 and 8 . Figure 8 shows normalized emittance $\varepsilon$ versus percent total negative ion beam intensity for each of the species. These data are derived from 10 or more measurements with standard deviations typically ranging from $3 \%$ to $6 \%$. The results of these measurements clearly exhibit a species-dependent effect. An analysis of the contributions by the various beam transport components in the test facility prior to emittance measurement such as given in Refs. 10 and 11, indicates that differences in energy spread are responsible for the observed differences in emittances of the respective ion beams.

The origin of the energy spread effect is thought to be attributable to differences in the intrinsic energy distributions associated with the sputter generation process. Thus, the results of these investigations reflect on the fundamental processes which occur during negative ion ejection (e.g., differences in momentum transfer, surface work function $\phi(\sigma)$, lattice binding energy $E_{b}$, and image potential $\left.V_{i}\right)$. Through these measurements, we also demonstrate the utility of high-resolution emittancemeasurement techniques for acquiring information concerning energy spreads within ion beams. 


\section{ACKNOWLEDGEMENTS}

The author expresses appreciation to Ms. Jeanette McBride for typing of the manuscript. 
References

1. G. D. Alton, Nucl. Instrum. and Meth. B37/38 (1989) 45.

2. G. D. Alton, to be published in IEEE Trans. Nucl. Sci.

3. V. E. Krohn, Jr., Appl. Phys. 38 (1962) 3523.

4. W. B. Herrmannsfeldt, SLAC Rep. No. 166 (1973).

5. J. H. Whealton, Nucl. Instrum. and Meth. 189 (1981) 55.

6. G. D. Alton and G. D. Mills, IEEE Trans. Nucl. Sci. NS-32 (1985) 1822.

7. G. D. Alton, J. W. McConnell, S. Tajima, and G. J. Nelson, Nucl. Instrum. and Meth. B24/25 (1987) 826.

8. G. D. Alton, Proc. 11th Symp. on Ion Sources and lon-Assisted Technology; Tokyo, Japan (1987) 157. G. D. Alton and J. W. McConnell, Nucl. Instrum. and Meth. A268 (1988) 445.

9. G. D. Alton, Nucl. Instrum. and Meth. A244 (1986) 133.

10. G. D. Alton and R. W. Sayer, J. of Phys. D: Appl. Phys. 22 (1989) 557.

11. G. D. Alton and R. W. Sayer, submitted for publication in Phys. Rev. B. 
Figure Captions

1. Calculated cesium positive ion trajectories in the source equipped with a spherical geometry cesium surface ionizer. Perveance: $P=2 \times 10^{-9}\left[\mathrm{AN}^{3 / 2}\right]$.

2. Calculated cesium positive ion current density distributions at the sputter sample surface of the source equipped with a spherical geometry cesium surface ionizer.

3. Calculated cesium positive ion trajectories in the source equipped with an ellipsoidal geometry cesium surface ionizer. Perveance: $P=17 \times 10^{-9}\left[\mathrm{AN}^{3 / 2}\right.$ ].

4. Calculated cesium positive ion current density distribution at the sputter sample surface of the source equipped with an ellipsoidal geometry ionizer.

5. Calculated cesium positive ion trajectories in the source equipped with a cylindrical geometry cesium surface ionizer. Perveance: $P=57 \times 10^{-9}$ [AN3/2].

6. Calculated cesium positive ion current density distribution at the sputter sample surface of the source equipped with a cylindrical geometry cesium surface ionizer.

7. Average normalized emittance $\varepsilon_{n}$ versus percentage average negative ion beam intensity for beams extracted from sources equipped with spherical, ellipsoidal, and cylindrical geometry cesium surface ionizers. Ion energy = $20 \mathrm{keV}$.

8. Normalized emittance $\varepsilon_{n}$ versus percentage ion beam intensity for $\boldsymbol{\Delta},{ }^{28} \mathrm{Si}^{-}(51$ $\mu \mathrm{A}) ; \square, 58 \mathrm{Ni}^{-}(13 \mu \mathrm{A}) ; \mathrm{O},{ }^{197} \mathrm{Au}-(41 \mu \mathrm{A})$ illustrating a species-dependent effect. Ion energy $=20 \mathrm{keV}$. 
ORNL-DWG 83-19379R

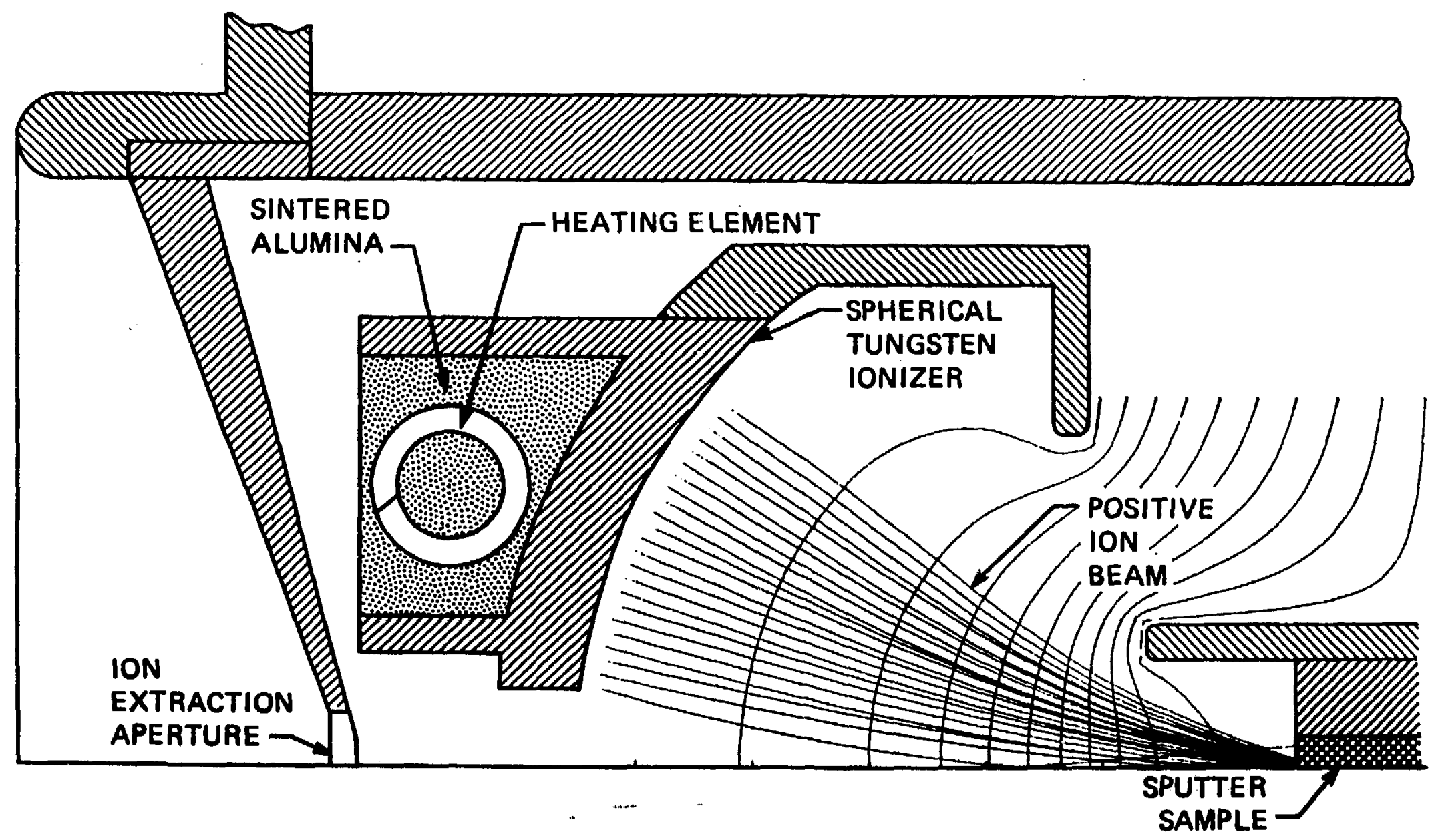

Fig. 1 


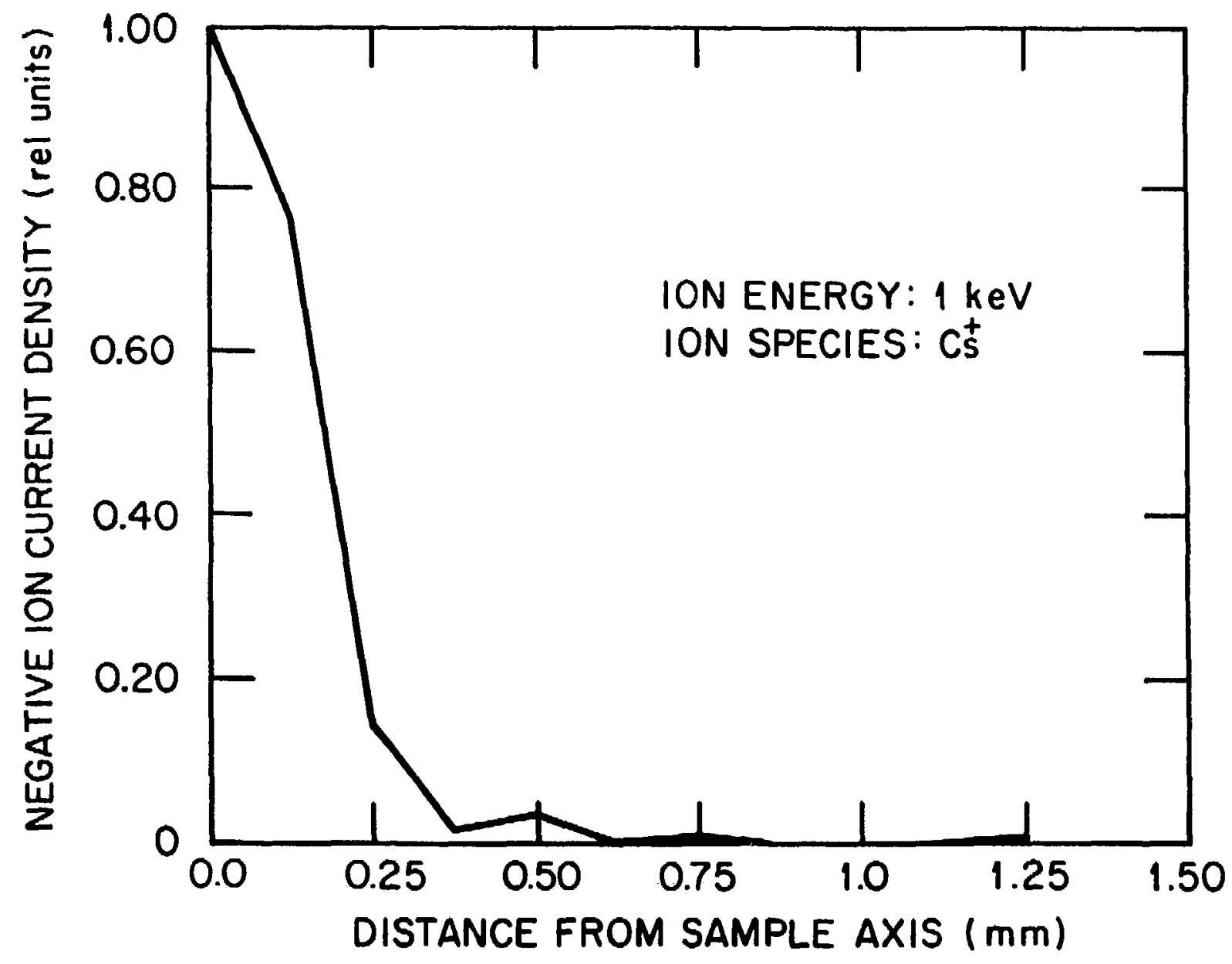

Fig. 2 




Fig. 3 
POSITIVE ION CURRENT DENSITY (rel. units)

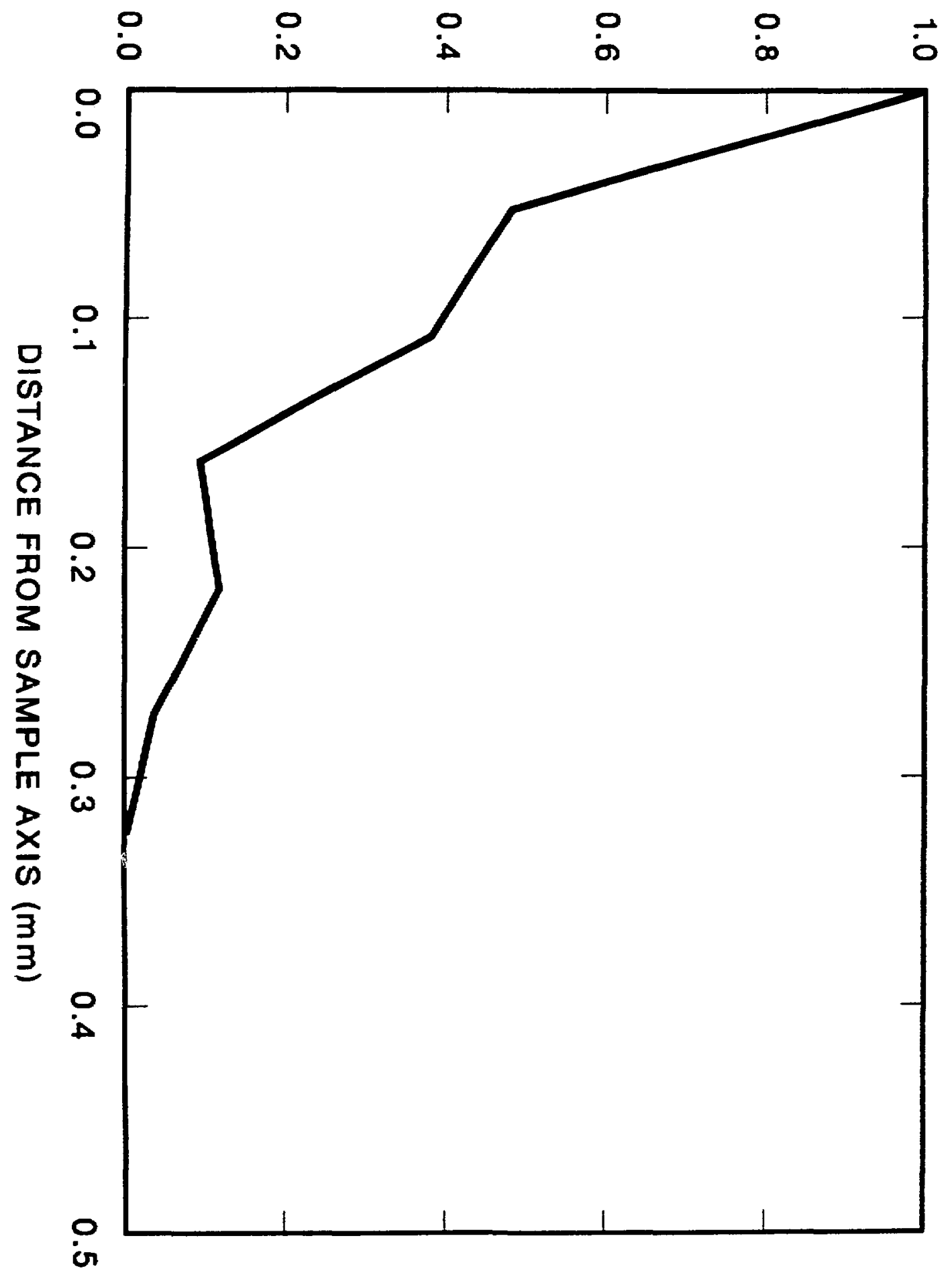

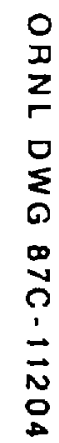




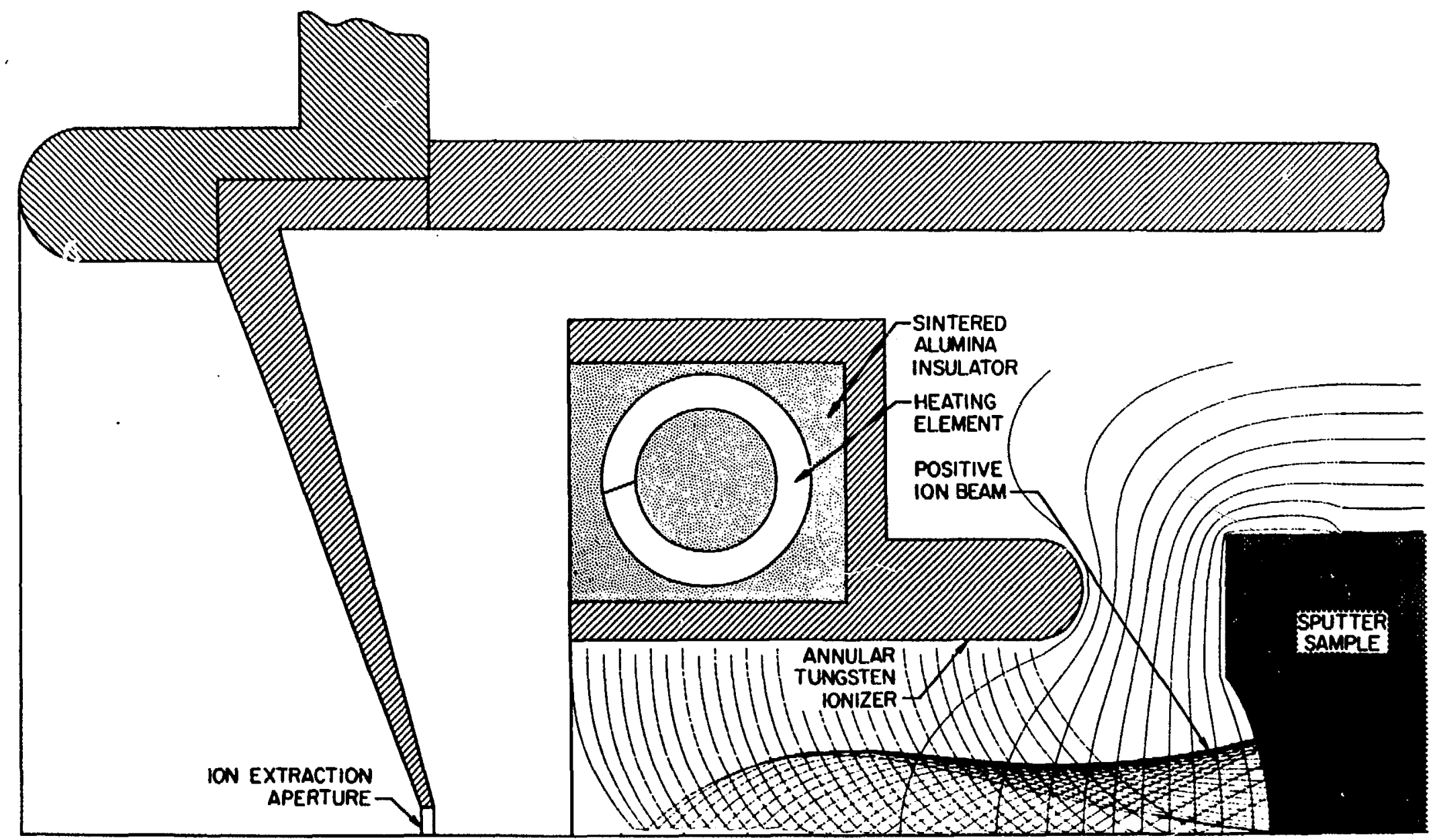

Fig. 5 


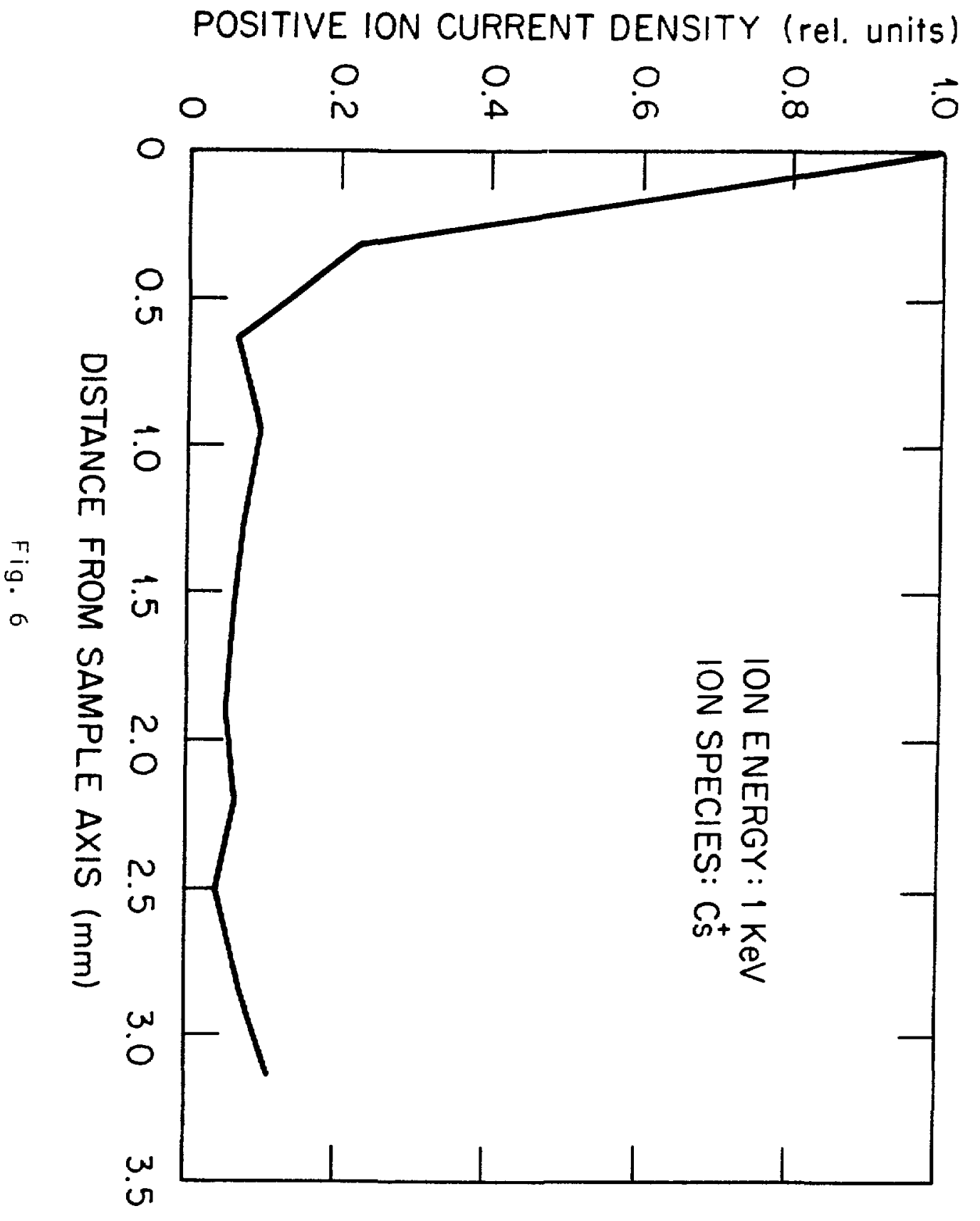

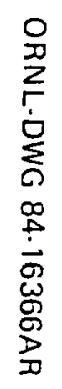




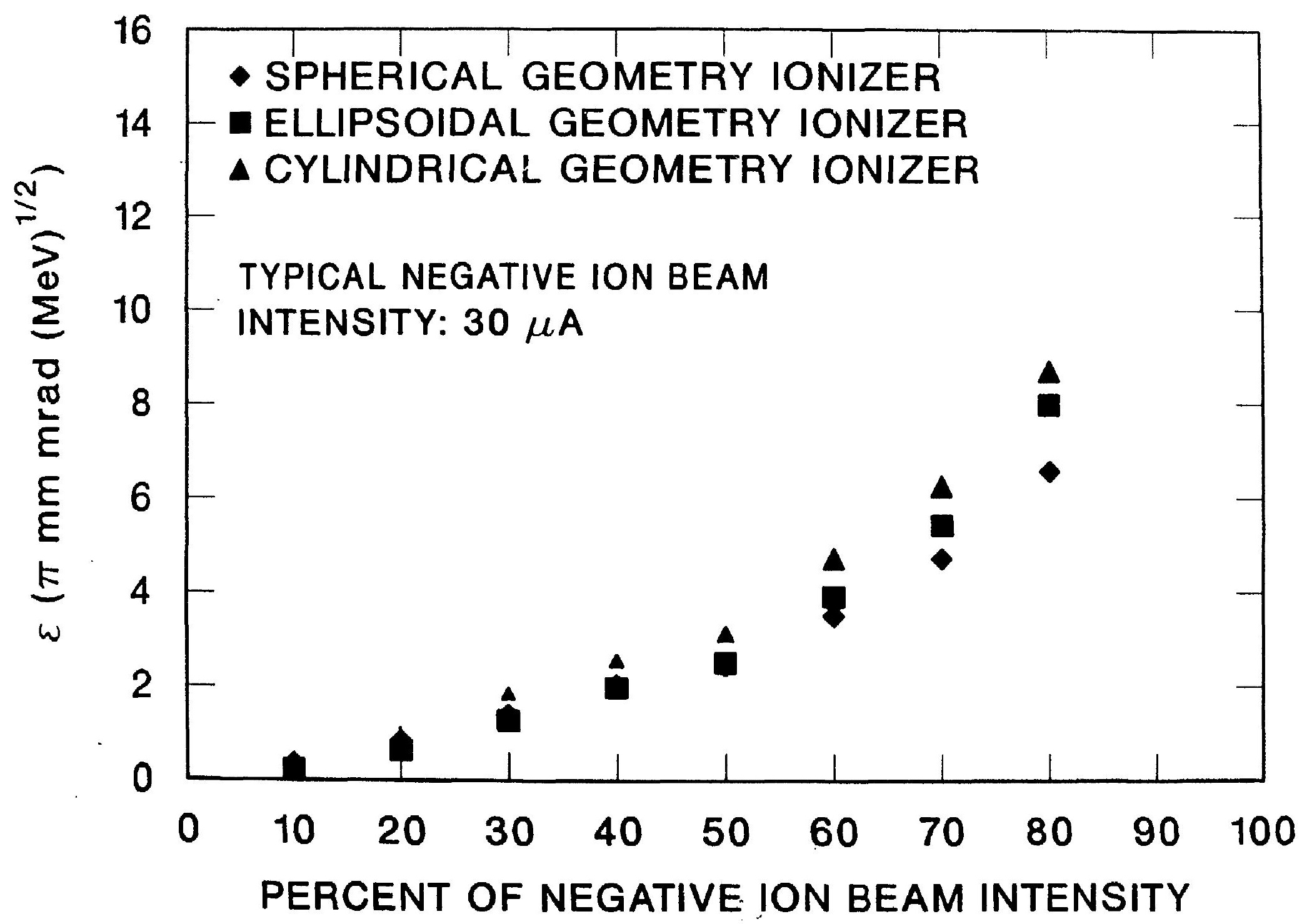




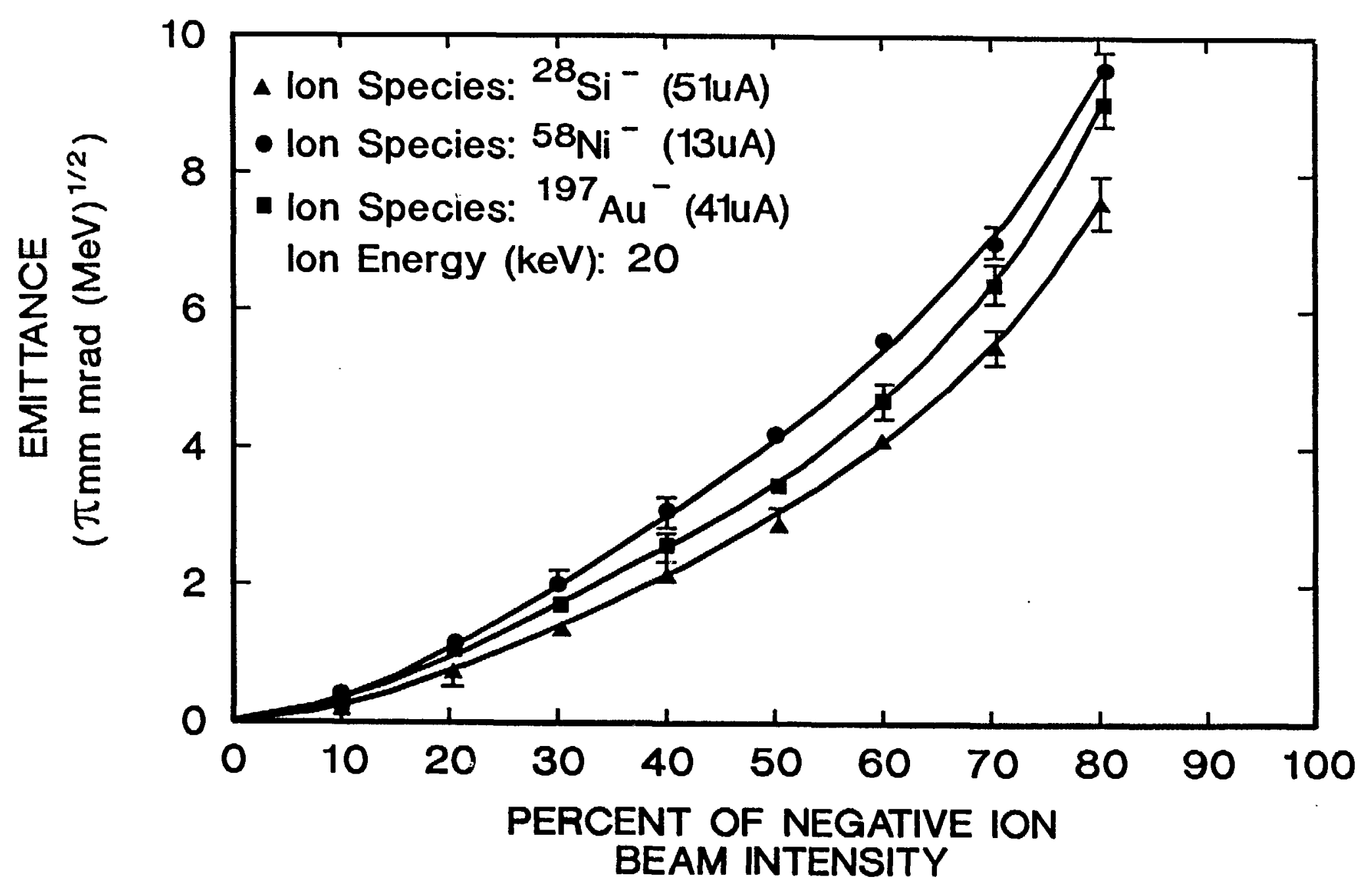

Fig. 8 